\title{
Computational modeling of creep in complex plane for reinforced materials
}

\author{
V. MONFARED* \\ Young Researchers and Elite Club, Zanjan Branch, Islamic Azad University, Zanjan, Iran
}

\begin{abstract}
Computational modeling for predicting the steady state creep behavior is presented in complex plane for reinforced materials by complex variable method. Both the fiber and matrix simultaneously creep at elevated temperatures and loading. We suppose that one dimension of the short fiber is small enough in comparison with the other two (see Fig. 1). In this formulation, plane stress state is used. Finally, displacement rate behaviors are predicted using compatibility, equilibrium, constitutive, and governing equations by complex variable method. One of the considerable applications of the method is in nano-composites analysis in elasticity or plasticity research.
\end{abstract}

Key words: creep, composite, complex plane.

\section{Introduction}

Creep is the inelastic response of materials loaded at high temperatures. Secondary stage creep or steady-state creep is a permanent state of creep, in which gradient and slope of the curve of $\dot{\varepsilon}-t$ and $\varepsilon-t$ is constant and positive, respectively, and ascending behavior is considered at this stage. All designs based on creep criterion must be carried out in this stage. Here, the creep strain rate reaches a minimum value and remains approximately constant over a relatively long period of time. An idealized creep curve shows the three stages during creep phenomenon (primary, secondary and tertiary). The secondary region (II) of the creep is characterized by steady-state (second stage) creep (the creep strain rate $\dot{\varepsilon}_{\text {min }}=\dot{\varepsilon}_{s s}$ is constant), in which competing mechanisms of strain hardening and recovery may be present. However, for engineering applications, the steady-state creep rate (plasticity) and elasticity are of major concern [1-22]. Polymer matrix composites (PMC's) are being increasingly employed in construction and their creep behaviors subjected to stress are receiving more research interest. For example, creep of PMC's used in spaceships is very dangerous and it may happen in spaceships. So the creep phenomenon must be analyzed to prevent some undesirable events. Complex variable method presents an important, simple, and applied tool for solving the problems in plasticity and elasticity subjects instead of analytical complex and intricate classic methods in other coordinates and systems. The present technique is also useful for cases involving anisotropic, thermoplastic, viscoplastic, plasticity, and creep problems.

Using complex variable methods enables many problems to be solved that would be nonflexible according to other schemes. Creep micro-deformations of the short fiber compos-

\footnotetext{
*e-mail: vahid_monfared_57@yahoo.com

Manuscript submitted 2016-09-20, revised 2017-03-10, initially accepted for publication 2017-05-28, published in December 2017.
}

ites are a significant characteristic of the materials which are under mechanical loads and different temperatures. Complex numbers are utilized in many scientific fields, including engineering and other sciences. In this work, the complex variables are used for determining the creep analysis in the short fiber composites in plane stress state for the anisotropic and orthotropic materials subjected to the applied load. The general and widespread texts on the complex variable method were developed by Muskhelishvili and Kolosov [1, 18], MilneThomson [2], Green and Zerna [3], and England [4]. In those works, complex variable method and theory was initially used and formulated in elasticity problems by Kolosov [18]. For instance, Gao [5] discussed both the displacement function and the Airy stress function methods which are based on an extended and complete version of Green's theory. Also, Barai and Weng [6] presented a micro-continuum modeling for the creep behavior of complex nanocrystalline materials. Moreover, micromechanical analysis of thermo-elasto-plastic behavior of the metal matrix composites was studied by Tang et al. [7]. In addition, interesting works have been wrtitten regarding the creep behaviors of materials [8-10]. Other mathematical models $[11,12]$ have been employed to analyze the steady state creep of the short fiber composites. These include stress behavior predictions using virtual fiber method at non-reinforced regions [11], and atomic properties [12]. Also, in recent years, some research works have been done and developed for analyzing the creep behavior by various and different methods [19-21]. In this paper, steady state creep behaviors of short fiber composites are studied analytically in the plane stress state by complex variable method. Creep displacement rate behaviors are predicted by means of the compatibility, equilibrium, constitutive, and governing equations using complex variable method. Finally, this paper presents a theoretical formulation to predict the creep behavior in complex plane for reinforced materials by complex variable method in the plasticity field. The solution and analysis of the creep problems is very intricate and complex in Cartesian or other coordinates. 


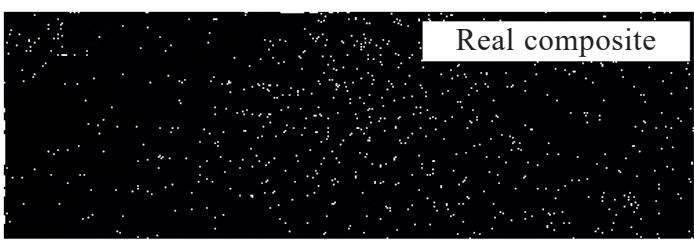

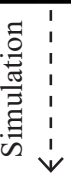
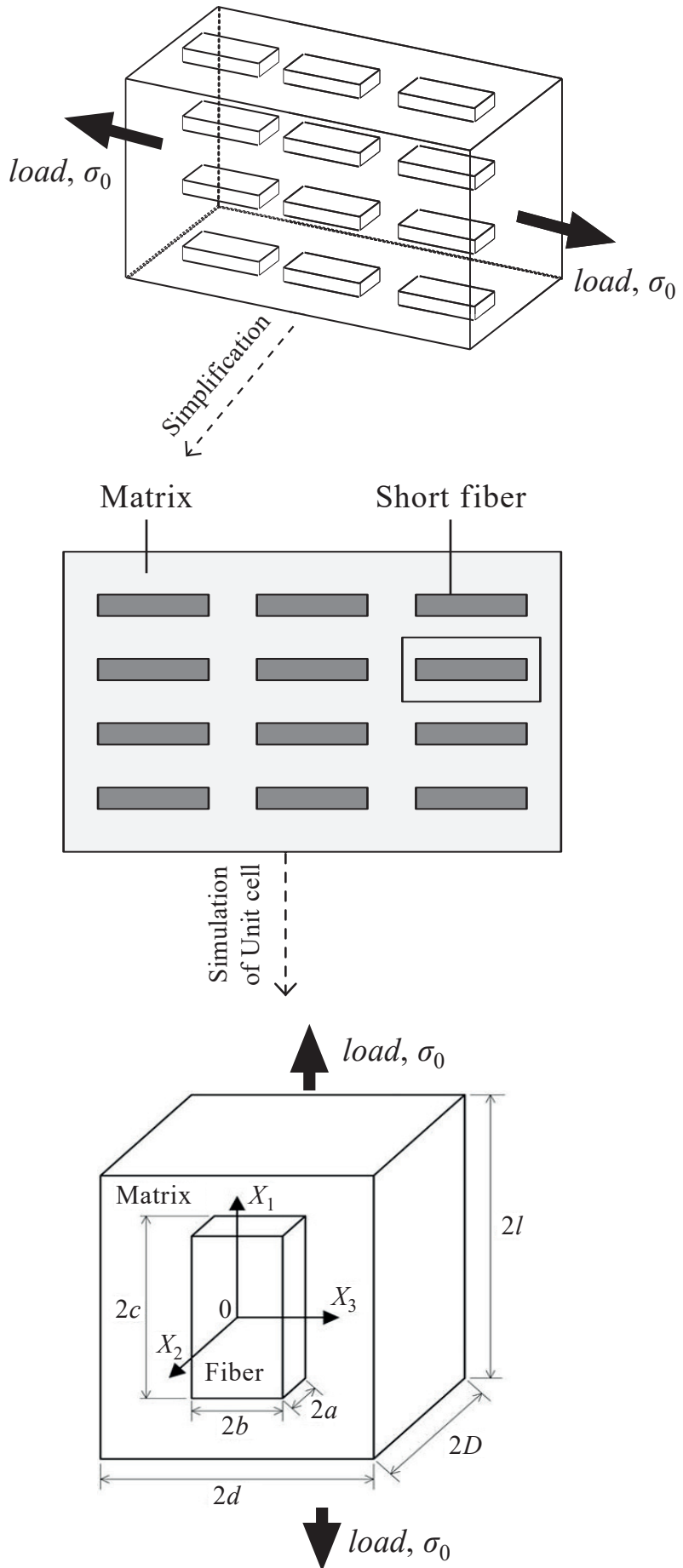

Fig. 1. A model of the unit cell with the creeping fiber and matrix under applied load (stress) " $\sigma_{0}$ " (in $\mathrm{X}_{1}$-direction)

\section{Material and method}

2.1. General explanation. Here, consider a plane stress state of the anisotropic unit cell (as a representative of a complete composite), nonlinearly plastic orthotropic material undergoing infinitesimal creep deformations in the usual Cartesian coordinate under applied loading (see Fig. 1).

Also, a unit cell is represented with a fiber and its surrounding matrix. These recent relations are only for simplification of the relations. The out-of-plane stress and strain components are considered, and $\dot{\varepsilon}_{33}$ is very small [13]. General shape of the unit cell with the creeping short fiber and matrix subjected to axial load in $\mathrm{X}_{1}$-direction is shown in Figs. 1, 2.

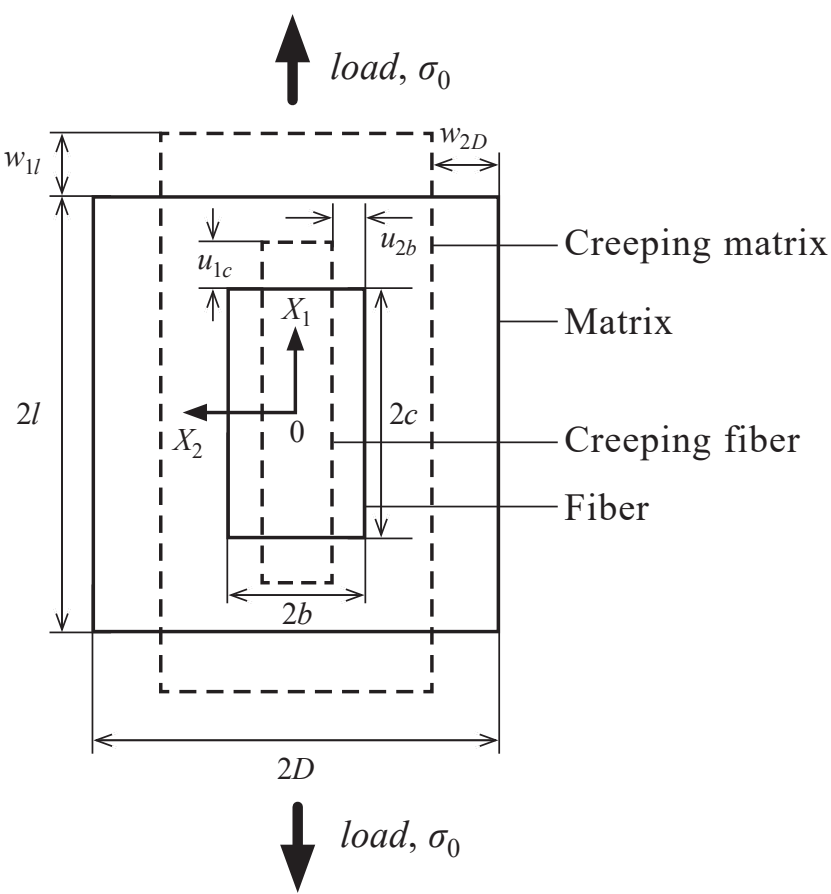

Fig. 2. Graphical explanation of the creeping fiber and matrix under tensile stress (load) " $\sigma_{0}$ " in $\mathrm{X}_{1}$-direction

Figure 1 shows the state before the creep, and Fig. 2 shows thestate after the creep. First of all, for plane stress problem, the compatibility equations $[1-4,13,22-25]$ reduce to the following conditions,

$$
\begin{aligned}
& \frac{\partial^{2} \dot{\varepsilon}_{33}}{\partial x_{2}^{2}}=0, \dot{\varepsilon}_{13}=\dot{\varepsilon}_{23}=0, \dot{\varepsilon}_{33} \neq 0 \\
& \frac{\partial^{2} \dot{\varepsilon}_{33}}{\partial x_{1}^{2}}=0, \dot{\varepsilon}_{13}=\dot{\varepsilon}_{23}=0, \dot{\varepsilon}_{33} \neq 0 \\
& \frac{\partial^{2} \dot{\varepsilon}_{33}}{\partial x_{1} \partial x_{2}}=0, \dot{\varepsilon}_{13}=\dot{\varepsilon}_{23}=0, \dot{\varepsilon}_{33} \neq 0
\end{aligned}
$$

$2 \frac{\partial^{2} \dot{\varepsilon}_{12}}{\partial x_{1} \partial x_{2}}-\frac{\partial^{2} \dot{\varepsilon}_{11}}{\partial x_{2}^{2}}-\frac{\partial^{2} \dot{\varepsilon}_{22}}{\partial x_{1}^{2}}=0, \dot{\varepsilon}_{13}=\dot{\varepsilon}_{23}=0, \dot{\varepsilon}_{33} \neq 0$. 
Now, the equilibrium equations, strain-displacement relations, incompressibility relation, and other basic equations (i.e. Eqs. (1) to (27)) are necessary to analyze the creep problem. In general, the equilibrium equations in absence of body forces are introduced as the following [1-4, 13, 23-25],

$$
\begin{gathered}
\frac{\partial \sigma_{11}}{\partial x_{1}}+\frac{\partial \sigma_{12}}{\partial x_{2}}+\frac{\partial \sigma_{13}}{\partial x_{3}}=0 \\
\frac{\partial \sigma_{21}}{\partial x_{1}}+\frac{\partial \sigma_{22}}{\partial x_{2}}+\frac{\partial \sigma_{23}}{\partial x_{3}}=0 \\
\frac{\partial \sigma_{31}}{\partial x_{1}}+\frac{\partial \sigma_{32}}{\partial x_{2}}+\frac{\partial \sigma_{33}}{\partial x_{3}}=0 \\
\sigma_{13}=\sigma_{23}=\sigma_{33}=0, \sigma_{12}\left(=\tau_{12}\right) \neq 0 .
\end{gathered}
$$

Also, the displacement components satisfy the following,

$$
\begin{gathered}
\sigma_{1}=\sigma_{11}=\sigma_{11}\left(x_{1}, x_{2}\right) \\
\sigma_{2}=\sigma_{22}=\sigma_{22}\left(x_{1}, x_{2}\right) \\
\sigma_{12}=\tau_{12}=\sigma_{12}\left(x_{1}, x_{2}\right) .
\end{gathered}
$$

Considering

$$
x \equiv x_{1}, y \equiv x_{2}, z \equiv x_{3}, c \gg b \gg a \text {. }
$$

In general, the strain rate-displacement rate relations are given by the following equations (linear Cauchy's equations written as time derivatives of strains and displacements in the steady state creep) $[1-4,13]$,

$$
\begin{gathered}
\dot{\varepsilon}_{11}=\dot{\varepsilon}_{1}=\frac{\partial \dot{u}_{1}}{\partial x_{1}} \\
\dot{\varepsilon}_{22}=\dot{\varepsilon}_{2}=\frac{\partial \dot{u}_{2}}{\partial x_{2}} \\
\dot{\varepsilon}_{33}=\dot{\varepsilon}_{3}=\frac{\partial \dot{u}_{3}}{\partial x_{3}} \\
\dot{\varepsilon}_{12}=\frac{1}{2} \dot{\gamma}_{12}=\frac{1}{2}\left(\frac{\partial \dot{u}_{1}}{\partial x_{2}}+\frac{\partial \dot{u}_{2}}{\partial x_{1}}\right) .
\end{gathered}
$$

In Fig. 2, $\left(u_{1}, u_{2}\right)$ and $\left(w_{1}, w_{2}\right)$ are displacement fields in the creeping fiber and matrix, respectively. Moreover, subscripts 1 and 2 are related to $x_{1}$ and $x_{2}$ directions; $u_{1 c}, u_{2 b}, w_{1 l}$ and $w_{2 D}$ are boundary values in the outer surfaces.

That is, the displacement rates $\left(\dot{u}_{1}, \dot{u}_{2}\right)$ are considered for the creeping fiber deformation rates. The generalized constitutive equations for the second stage creep deformations of the fiber and matrix in $x_{1}, x_{2}$ and $x_{3}$ directions are obtained by combination of Eqs. (1-36). So we have

$$
\tau_{1}=\frac{\sigma_{2}-\sigma_{3}}{2}, \sigma_{1}>\sigma_{2}>\sigma_{3}, \dot{\varepsilon}_{1}>\dot{\varepsilon}_{2}>\dot{\varepsilon}_{3}
$$

$$
\begin{aligned}
& \tau_{2}=\frac{\sigma_{3}-\sigma_{1}}{2}, \sigma_{1}>\sigma_{2}>\sigma_{3}, \quad \dot{\varepsilon}_{1}>\dot{\varepsilon}_{2}>\dot{\varepsilon}_{3} \\
& \tau_{3}=\frac{\sigma_{1}-\sigma_{2}}{2}, \sigma_{1}>\sigma_{2}>\sigma_{3}, \quad \dot{\varepsilon}_{1}>\dot{\varepsilon}_{2}>\dot{\varepsilon}_{3},
\end{aligned}
$$

where $\sigma_{1}, \sigma_{2}$ and $\sigma_{3}$ are the principal stresses and $\tau_{1}, \tau_{2}$ and $\tau_{3}$ are the principal shear stress. All relations are the same for creeping fiber and matrix (we can derive from the related parameters because the creep state is steady and permanent or second stage creep). Also $\dot{\gamma}_{1}, \dot{\gamma}_{2}$ and $\dot{\gamma}_{3}$ are the principal shear strain rates and $\dot{\varepsilon}_{1}, \dot{\varepsilon}_{2}$ and $\dot{\varepsilon}_{3}$ are the principal strain rates.

$$
\begin{aligned}
& \dot{\gamma}_{1}=\dot{\varepsilon}_{2}-\dot{\varepsilon}_{3} \\
& \dot{\gamma}_{2}=\dot{\varepsilon}_{3}-\dot{\varepsilon}_{1} \\
& \dot{\gamma}_{3}=\dot{\varepsilon}_{1}-\dot{\varepsilon}_{2} .
\end{aligned}
$$

In the following, hydrostatic and equivalent Huber-Mises stresses are given by [1-4, 13],

$$
\begin{gathered}
\sigma_{H}=\frac{\left(\sigma_{1}+\sigma_{2}+\sigma_{3}\right)}{3} \\
\sigma_{e}=\sqrt{2}\left(\tau_{1}{ }^{2}+\tau_{2}{ }^{2}+\tau_{3}{ }^{2}\right)^{\frac{1}{2}} .
\end{gathered}
$$

In addition, equivalent strain rate is $[1-4,13]$,

$$
\dot{\varepsilon}_{e}=\frac{\sqrt{2}}{3}\left(\dot{\gamma}_{1}^{2}+\dot{\gamma}_{2}^{2}+\dot{\gamma}_{3}^{2}\right)^{\frac{1}{2}} \text {. }
$$

Equation (26) is the incompressibility condition.

$$
\frac{\partial \dot{u}_{1}}{\partial x_{1}}+\frac{\partial \dot{u}_{2}}{\partial x_{2}}+\frac{\partial \dot{u}_{3}}{\partial x_{3}}=\dot{\varepsilon}_{1}+\dot{\varepsilon}_{2}+\dot{\varepsilon}_{3}=0
$$

In this paper, the steady state creep problem in composites is studied for two cases (constant $€$ and variable $€$ ) by complex variable method. According to the relationship between the shear stress and the shear strain rate $(\tau \propto \dot{\gamma})$, have

$$
\frac{\dot{\gamma}_{1}}{\tau_{1}}+\frac{\dot{\gamma}_{2}}{\tau_{2}}+\frac{\dot{\gamma}_{3}}{\tau_{3}}=€(€ \text { is a constant or variable parameter }) .
$$

The steady state creep phenomenon mainly happens in the polymeric matrix composites (PMCs) at low stresses and temperatures [14-17].

Therefore, parameter $€$ is supposed to be constant. It should be mentioned that parameter $€$ varies in high stress and temperature regimes because the steady state creep behavior of PMCs is exceedingly nonlinear and fairly unclear in high stresses and temperatures.

Thus, the presented method is applicable for the reinforced materials such as polymeric matrix composites (PMCs) at different conditions. The combination of Eqs. (23-27) yields

$$
\dot{\varepsilon}_{1}=\frac{€}{2}\left(\sigma_{1}-\frac{\left(\sigma_{1}+\sigma_{2}+\sigma_{3}\right)}{3}\right)
$$




$$
\begin{aligned}
& \dot{\varepsilon}_{2}=\frac{€}{2}\left(\sigma_{2}-\frac{\left(\sigma_{1}+\sigma_{2}+\sigma_{3}\right)}{3}\right) \\
& \dot{\varepsilon}_{3}=\frac{€}{2}\left(\sigma_{3}-\frac{\left(\sigma_{1}+\sigma_{2}+\sigma_{3}\right)}{3}\right) .
\end{aligned}
$$

Consequently, parameter $€$ may be given by the following,

$$
€=\frac{3 \dot{\varepsilon}_{e}}{\sigma_{e}} .
$$

We know that the equivalent strain rate $\dot{\varepsilon}_{e}$ have a direct relation with time $\Omega(t)$ and equivalent stress $\Gamma\left(\sigma_{e}\right)$, that is,

$$
\dot{\varepsilon}_{e}=\mathcal{A} \Omega(t) \Gamma\left(\sigma_{e}\right) .
$$

Parameter $A$ is a constant for obtaining the creep constitutive equations.

$$
\dot{\varepsilon}_{e}=\Omega(t) \Gamma\left(\sigma_{e}\right), \text { considering } A=1 \text {. }
$$

This process of the solution (Eqs. $(32,33)$ is similar to (resulted from) the separation of variables like the methods used in engineering mathematics. So

$$
\begin{aligned}
& \dot{\varepsilon}_{1}=\frac{3 \Omega(t) \Gamma\left(\sigma_{e}\right)}{2 \sigma_{e}}\left(\sigma_{1}-\frac{\left(\sigma_{1}+\sigma_{2}+\sigma_{3}\right)}{3}\right) \\
& \dot{\varepsilon}_{2}=\frac{3 \Omega(t) \Gamma\left(\sigma_{e}\right)}{2 \sigma_{e}}\left(\sigma_{2}-\frac{\left(\sigma_{1}+\sigma_{2}+\sigma_{3}\right)}{3}\right) \\
& \dot{\varepsilon}_{3}=\frac{3 \Omega(t) \Gamma\left(\sigma_{e}\right)}{2 \sigma_{e}}\left(\sigma_{3}-\frac{\left(\sigma_{1}+\sigma_{2}+\sigma_{3}\right)}{3}\right) .
\end{aligned}
$$

Plane stress state must be considered for solving Eqs. (34-36), thus, solution of the mentioned equations yields,

$$
\begin{aligned}
& \sigma_{1}=\frac{2}{3}\left(\frac{\Gamma^{-1}\left(\frac{\dot{\varepsilon}_{e}}{\Omega(t)}\right)}{\dot{\varepsilon}_{e}}\right)\left(2 \dot{\varepsilon}_{1}+\dot{\varepsilon}_{2}\right) \\
& \sigma_{2}=\frac{2}{3}\left(\frac{\Gamma^{-1}\left(\frac{\dot{\varepsilon}_{e}}{\Omega(t)}\right)}{\dot{\varepsilon}_{e}}\right)\left(2 \dot{\varepsilon}_{2}+\dot{\varepsilon}_{1}\right) \\
& \sigma_{2}=0
\end{aligned}
$$

Eqs. (37-39) are determined by combination of the Eqs. (1-36). The simple forms of Eqs. (37-39) are as the following,

$$
\begin{aligned}
& \sigma_{1}=\frac{4 \dot{\varepsilon}_{1} \sigma_{\mathrm{e}}+2 \dot{\varepsilon}_{2} \sigma_{\mathrm{e}}}{3 \dot{\varepsilon}_{\mathrm{e}}}=\frac{4 \dot{\varepsilon}_{1}+2 \dot{\varepsilon}_{2}}{€} \\
& \sigma_{2}=\frac{2 \dot{\varepsilon}_{1} \sigma_{\mathrm{e}}+4 \dot{\varepsilon}_{2} \sigma_{\mathrm{e}}}{3 \dot{\varepsilon}_{\mathrm{e}}}=\frac{2 \dot{\varepsilon}_{1}+4 \dot{\varepsilon}_{2}}{€} \\
& \sigma_{3}=0 \\
& \sigma_{12}=\frac{\dot{\gamma}_{12} \sigma_{e}}{3 \dot{\varepsilon}_{\mathrm{e}}}=\frac{\dot{\gamma}_{12}}{€} .
\end{aligned}
$$

Where $\dot{\varepsilon}_{1}, \dot{\varepsilon}_{2}, \dot{\varepsilon}_{3}$ and $\dot{\varepsilon}_{12}$ are the strain rate components in the directions indicated by subscripts. Parameters of $\sigma_{1}, \sigma_{2}, \sigma_{3}$ and $\tau_{12}$ are the stress components in the directions indicated by subscripts. Now, problem solution method is divided to the two different sections of part 1 (constant $€$ ) and part 2 (variable $€$ ). It should be mentioned that in above formulations, equivalent strain rate is introduced by the following,

$$
\dot{\varepsilon}_{e}=\dot{\varepsilon}_{e}\left(\dot{\varepsilon}_{1}, \dot{\varepsilon}_{2}, \dot{\varepsilon}_{3}, \sigma_{1}, \sigma_{2}, \sigma_{3}, t\right) .
$$

2.2. Part 1: constant $€$. For constant $€$, the stress and temperature values are low. Using Eqs. (40-43), (5-8), and considering compatibility relations presented in Eqs. (1-4) along with Eqs. (13-16), yields,

$$
\begin{aligned}
& 4 \frac{\partial^{2} \dot{u}_{1}}{\partial x_{1}^{2}}+3 \frac{\partial^{2} \dot{u}_{2}}{\partial x_{1} \partial x_{2}}+\frac{\partial^{2} \dot{u}_{2}}{\partial x_{2}^{2}}=0 \\
& \frac{\partial^{2} \dot{u}_{2}}{\partial x_{1}^{2}}+3 \frac{\partial^{2} \dot{u}_{1}}{\partial x_{1} \partial x_{2}}+4 \frac{\partial^{2} \dot{u}_{2}}{\partial x_{2}^{2}}=0 .
\end{aligned}
$$

Equations $(45,46)$ must be satisfied by the following compatibility equation of Eq. (47),

$$
\frac{\partial^{2}\left(\frac{\partial \dot{u}_{1}}{\partial x_{2}}+\frac{\partial \dot{u}_{2}}{\partial x_{1}}\right)}{\partial x_{1} \partial x_{2}}-\frac{\partial^{2}\left(\frac{\partial \dot{u}_{1}}{\partial x_{1}}\right)}{\partial x_{2}^{2}}-\frac{\partial^{2}\left(\frac{\partial \dot{u}_{2}}{\partial x_{2}}\right)}{\partial x_{1}^{2}}=0 .
$$

This system of equations (Eqs. (45-47)) can be solved either concurrently or individually.

$$
x_{1}=\frac{\bar{z}}{2}+\frac{z}{2}, x_{2}=\frac{\bar{z}}{2} i-\frac{z}{2} i
$$

In which,

$$
z=x_{1}+i x_{2}, \quad \bar{z}=x_{1}-i x_{2}, i^{2}=-1 .
$$

This gives, with the use of the chain rule $[1-4,13]$,

$$
\begin{aligned}
\frac{\partial}{\partial z} & =\frac{1}{2}\left(\frac{\partial}{\partial x_{1}}-i \frac{\partial}{\partial x_{2}}\right), \frac{\partial}{\partial x_{1}}=\frac{\partial}{\partial z}+\frac{\partial}{\partial \bar{z}}, \frac{\partial^{2}}{\partial x_{1}{ }^{2}}= \\
& =\frac{\partial^{2}}{\partial z^{2}}+\frac{\partial^{2}}{\partial \bar{z}^{2}}+2 \frac{\partial^{2}}{\partial z \partial \bar{z}}, \frac{\partial}{\partial x_{2}}=i\left(\frac{\partial}{\partial z}-\frac{\partial}{\partial \bar{z}}\right) \\
\frac{\partial}{\partial \bar{z}} & =\frac{1}{2}\left(\frac{\partial}{\partial x_{1}}+i \frac{\partial}{\partial x_{2}}\right), \frac{\partial^{2}}{\partial x_{2}{ }^{2}}=-\left(\frac{\partial^{2}}{\partial z^{2}}+\frac{\partial^{2}}{\partial \bar{z}^{2}}-\right. \\
& \left.-2 \frac{\partial^{2}}{\partial z \partial \bar{z}}\right), \frac{\partial^{2}}{\partial x_{1} \partial x_{2}}=i\left(\frac{\partial^{2}}{\partial z^{2}}-\frac{\partial^{2}}{\partial \bar{z}^{2}}\right) .
\end{aligned}
$$

Substituting Eqs. $(13,14)$ and $(50,51)$ into Eqs. (45-47) with considering Eqs. (40-43) will lead to 


$$
\begin{gathered}
\left(\frac{\partial \dot{u}_{1}}{\partial z}+\frac{\partial \dot{u}_{1}}{\partial \bar{z}}\right)\left(\sigma_{1}-2 \sigma_{2}\right)+ \\
+i\left(\frac{\partial \dot{u}_{2}}{\partial z}-\frac{\partial \dot{u}_{2}}{\partial \bar{z}}\right)\left(2 \sigma_{1}-\sigma_{2}\right)=0 \\
\left(\frac{\partial^{2}\left[\left(-\frac{\partial \dot{u}_{1}}{\partial z}+\frac{\partial \dot{u}_{1}}{\partial \bar{z}}\right)+i\left(-\frac{\partial \dot{u}_{2}}{\partial z}+\frac{\partial \dot{u}_{2}}{\partial \bar{z}}\right)\right]}{\partial z^{2}}-\right. \\
\left.-\frac{\partial^{2}\left[\left(-\frac{\partial \dot{u}_{1}}{\partial z}+\frac{\partial \dot{u}_{1}}{\partial \bar{z}}\right)+i\left(\frac{\partial \dot{u}_{2}}{\partial z}+\frac{\partial \dot{u}_{2}}{\partial \bar{z}}\right)\right]}{\partial z^{2}}\right)+ \\
+\left(\frac{\partial^{2}\left(\frac{\partial \dot{u}_{1}}{\partial z}+\frac{\partial \dot{u}_{1}}{\partial \bar{z}}\right)}{\partial z^{2}}+\frac{\partial^{2}\left(\frac{\partial \dot{u}_{1}}{\partial z}+\frac{\partial \dot{u}_{1}}{\partial \bar{z}}\right)}{\partial \bar{z}^{2}}-2 \frac{\partial^{2}\left(\frac{\partial \dot{u}_{2}}{\partial z}+\frac{\partial \dot{u}_{2}}{\partial \bar{z}}\right)}{\partial z \partial \bar{z}}\right)- \\
+i\left(\frac{\partial^{2}\left(\frac{\partial \dot{u}_{2}}{\partial z}-\frac{\partial \dot{u}_{2}}{\partial \bar{z}}\right)}{\partial z^{2}}+\frac{\partial^{2}\left(\frac{\partial \dot{u}_{2}}{\partial z}+\frac{\partial \dot{u}_{2}}{\partial \bar{z}}\right)}{\partial \bar{z}^{2}}+\right. \\
\left.+2 \frac{\partial^{2}\left(\frac{\partial \dot{u}_{2}}{\partial z}+\frac{\partial \dot{u}_{2}}{\partial \bar{z}}\right)}{\partial z \partial \bar{z}}\right)=0
\end{gathered}
$$

Substituting Eqs. (45-47) into Eqs. (50,51) for constant $€$, will lead to

$$
\begin{aligned}
& 3\left[\frac{\partial^{2} \dot{u}_{1}}{\partial z^{2}}+\frac{\partial^{2} \dot{u}_{1}}{\partial \bar{z}^{2}}+i\left(\frac{\partial^{2} \dot{u}_{2}}{\partial z^{2}}-\frac{\partial^{2} \dot{u}_{2}}{\partial \bar{z}^{2}}\right)\right]+10 \frac{\partial^{2} \dot{u}_{1}}{\partial z \partial \bar{z}}=0 \\
& -3\left[\frac{\partial^{2} \dot{u}_{2}}{\partial z^{2}}+\frac{\partial^{2} \dot{u}_{2}}{\partial \bar{z}^{2}}-i\left(\frac{\partial^{2} \dot{u}_{1}}{\partial z^{2}}-\frac{\partial^{2} \dot{u}_{1}}{\partial \dot{z}^{2}}\right)\right]+10 \frac{\partial^{2} \dot{u}_{2}}{\partial z \partial \bar{z}}=0
\end{aligned}
$$

$$
\begin{aligned}
& \left(\frac{\partial^{2}\left[\left(-\frac{\partial \dot{u}_{1}}{\partial z}+\frac{\partial \dot{u}_{1}}{\partial \bar{z}}\right)+i\left(\frac{\partial \dot{u}_{2}}{\partial z}+\frac{\partial \dot{u}_{2}}{\partial \bar{z}}\right)\right]}{\partial z^{2}}-\right. \\
& \left.-\frac{\partial^{2}\left[\left(-\frac{\partial \dot{u}_{1}}{\partial z}+\frac{\partial \dot{u}_{1}}{\partial \bar{z}}\right)+i\left(\frac{\partial \dot{u}_{2}}{\partial z}+\frac{\partial \dot{u}_{2}}{\partial \bar{z}}\right)\right]}{\partial \bar{z}^{2}}\right)+ \\
& +\left(\frac{\partial^{2}\left(\frac{\partial \dot{u}_{1}}{\partial z}+\frac{\partial \dot{u}_{1}}{\partial \bar{z}}\right)}{\partial z^{2}}+\frac{\partial^{2}\left(\frac{\partial \dot{u}_{1}}{\partial z}+\frac{\partial \dot{u}_{1}}{\partial \bar{z}}\right)}{\partial \bar{z}^{2}}-2 \frac{\partial^{2}\left(\frac{\partial \dot{u}_{1}}{\partial z}+\frac{\partial \dot{u}_{1}}{\partial \bar{z}}\right)}{\partial z \partial \bar{z}}\right)- \\
& -i\left(\frac{\partial^{2}\left(\frac{\partial \dot{u}_{2}}{\partial z}-\frac{\partial \dot{u}_{2}}{\partial \bar{z}}\right)}{\partial z^{2}}+\frac{\partial^{2}\left(\frac{\partial \dot{u}_{2}}{\partial z}-\frac{\partial \dot{u}_{2}}{\partial \bar{z}}\right)}{\partial \bar{z}^{2}}+\right. \\
& \left.+2 \frac{\partial^{2}\left(\frac{\partial \dot{u}_{2}}{\partial z}-\frac{\partial \dot{u}_{2}}{\partial \bar{z}}\right)}{\partial z \partial \bar{z}}\right)=0 .
\end{aligned}
$$

Adding and subtracting of Eqs. $(54,55)$ yields,

$$
\begin{aligned}
& 3\left[(1+i) \frac{\partial^{2} U}{\partial z^{2}}+(1-i) \frac{\partial^{2} \bar{U}}{\partial \bar{z}^{2}}\right]+ \\
& +5\left[(1-i) \frac{\partial^{2} U}{\partial z \partial \bar{z}}+(1+i) \frac{\partial^{2} \bar{U}}{\partial z \partial \bar{z}}\right]=0
\end{aligned}
$$

$$
\begin{aligned}
& 3\left[(1-i) \frac{\partial^{2} U}{\partial z^{2}}+(1+i) \frac{\partial^{2} \bar{U}}{\partial \bar{z}^{2}}\right]+ \\
& +5\left[(1+i) \frac{\partial^{2} U}{\partial z \partial \bar{z}}+(-i+1) \frac{\partial^{2} \bar{U}}{\partial z \partial \bar{z}}\right]=0 .
\end{aligned}
$$

Where $U=\dot{u}_{1}+i \dot{u}_{2}, \bar{U}=\dot{u}_{1}-i \dot{u}_{2}, \dot{u}_{1}=\frac{U+\bar{U}}{2}, \dot{u}_{2}=\frac{U-\bar{U}}{2 i}$, So have

$$
\begin{aligned}
\dot{u}_{1}+\dot{u}_{2}= & \frac{i U+U-\bar{U}+i \bar{U}}{2 i} \\
\dot{u}_{1}-\dot{u}_{2} & =\frac{i U+U-\bar{U}+i \bar{U}}{2 i} .
\end{aligned}
$$

With simplification of Eqs. $(57,58)$ and considering compatibility equations (Eqs. (1-4)), we have

$$
\begin{gathered}
\frac{\partial^{2} \bar{U}}{\partial \bar{z}^{2}}=-\frac{(1+i)}{(1-i)} \frac{\partial^{2} U}{\partial z^{2}}-\frac{5}{3}\left[\frac{\partial^{2} U}{\partial z \partial \bar{z}}+\right. \\
\left.+\frac{(1+i)}{(1-i)} \frac{\partial^{2} \bar{U}}{\partial z \partial \bar{z}}\right]+\theta(z, \bar{z}) \\
10 i \frac{\partial^{2} \bar{U}}{\partial z \partial \bar{z}}=-\theta(z, \bar{z}) .
\end{gathered}
$$

In (61-62), the parameter of $\theta(z, \bar{z})$ arises from the effect of compatibility equations. Integrating Eq. (62) with respect to $\bar{z}, z$ gives

$$
\bar{U}=\dot{u}_{1}-i \dot{u}_{2}=\oint_{c} \varnothing(z) d z+\rho(z)+\varpi(z, \bar{z}),
$$

where $c$ is closed finite and defined region in the steady state creep.

$$
\oint_{c} \varnothing(z) d z=\left.2 \pi i \operatorname{Res} \varnothing(z)\right|_{z=z_{0}} .
$$

That is,

$$
\begin{aligned}
& \dot{u}_{1}=\rho(z) \\
& \dot{u}_{2}=\varpi(z, \bar{z})-\left.2 \pi \operatorname{Res} \varnothing(z)\right|_{z=z_{0}} \\
& U=\dot{u}_{1}+i \dot{u}_{2}=\rho(z)+i\left(\varpi(z, \bar{z})-\left.2 \pi \operatorname{Res} \varnothing(z)\right|_{z=z_{0}}\right) .
\end{aligned}
$$

Also $\rho(z), \varnothing(z), U, \bar{U}$ and $\varpi(z, \bar{z})$ are analytic functions. Next, boundary conditions must be satisfied by the obtained displacement rate presented in Eqs. (68-75). For example $\varnothing(z)$ may be assumed to a polynomial function in order of $n$ with its unknown coefficients, and these unknowns can be determined by the following boundary conditions (Eqs. (68-75)).

$$
\begin{aligned}
& \dot{u}_{1}\left(0, x_{2}\right)=-\dot{u}_{1}\left(-c, x_{2}\right)=\dot{u}_{f T} \\
& \dot{u}_{1}\left(l-\frac{c}{2}, x_{2}\right)=-\dot{u}_{1}\left(c-\frac{l}{2}, x_{2}\right)=\dot{u}_{1 m T}
\end{aligned}
$$




$$
\begin{aligned}
& \dot{u}_{2}\left(x_{1}, \frac{-D}{2}\right)=-\dot{u}_{2}\left(x_{1}, \frac{D}{2}\right)=\dot{u}_{2 m L} \\
& \dot{u}_{2}\left(x_{1}, \frac{-b}{2}\right)=-\dot{u}_{2}\left(x_{1}, \frac{b}{2}\right)=\dot{u}_{2 f L} \\
& \dot{u}_{1}\left(0, x_{2}\right)=\dot{u}_{2}\left(x_{1}, 0\right)=0 \\
& \dot{u}_{2}\left(x_{1},-b\right)=\dot{u}_{2}\left(x_{1}, b\right)=\frac{b}{c} \dot{u}_{1 c} \\
& \dot{u}_{1}\left(c, x_{2}\right)=\dot{u}_{1}\left(-c, x_{2}\right)=\frac{c}{b} \dot{u}_{2 b} \\
& \dot{u}_{1_{x}}=\dot{u}_{2 y}, \dot{u}_{1_{y}}=\dot{u}_{2_{x}} .
\end{aligned}
$$

In boundary conditions (68-75), the subscript of $f, T$ and $m$ indicate to the fiber, top, matrix respectively.

2.3. Part 2: variable $€$. Considering variable $€$ is related to the high stress and temperature values. With assumption of variable $€$ compatibility equation, Eq. (1-4), and using Eqs. $(24,25)$ and Eqs. $(40,41)$, have

$$
\begin{gathered}
\frac{\sigma_{1}^{2}}{\left(2 \frac{\partial \dot{u}_{1}}{\partial x_{1}}+\frac{\partial \dot{u}_{2}}{\partial x_{2}}\right)^{2}}-\frac{\sigma_{2}^{2}}{\left(\frac{\partial \dot{u}_{1}}{\partial x_{1}}+\frac{\partial \dot{u}_{2}}{\partial x_{2}}\right)^{2}}=0 \\
\frac{\partial^{2}\left(\frac{\partial \dot{u}_{1}}{\partial x_{2}}+\frac{\partial \dot{u}_{2}}{\partial x_{1}}\right)}{\partial x_{1} \partial x_{2}}-\frac{\partial^{2}\left(\frac{\partial \dot{u}_{1}}{\partial x_{1}}\right)}{\partial x_{2}^{2}}-\frac{\partial^{2}\left(\frac{\partial \dot{u}_{2}}{\partial x_{2}}\right)}{\partial x_{1}^{2}}=0 .
\end{gathered}
$$

Equation (76) indicates to the dependency of $\sigma_{1}$ and $\sigma_{2}$ together in the plane stress state. Now, equation of the creep rates with variable $€$ is obtained by the following solution method,

$$
\begin{aligned}
& \left(\frac{\partial \dot{u}_{1}}{\partial z}-\frac{\partial \dot{u}_{1}}{\partial \bar{z}}\right)=0 \\
& \left(\frac{\partial \dot{u}_{2}}{\partial z}-\frac{\partial \dot{u}_{2}}{\partial \bar{z}}\right)=0 .
\end{aligned}
$$

Solving Eqs. $(78,79)$ simultaneously yields

$$
\Psi=z-z_{1} \bar{z}, \quad \mathbf{C}^{*}=z
$$

Where $z_{1}, z$ and $\bar{z}$ are the complex variables. With above assumptions (Eq. (80)) and considering compatibility equations (Eqs. (1-4)), we obtain

$$
\dot{u}_{1}=\dot{u}_{1}\left(z-z_{1} \bar{z}\right)+\mp(z, \bar{z})+\mathbf{3}(z) .
$$

Also for $\dot{u}_{2}$,

$$
\dot{u}_{2}=\dot{u}_{2}(z)+\text { Y }(z, \bar{z})
$$

In (81-82), $\mp(z, \bar{z}), \mathbf{3}(z)$ and $y(z, \bar{z})$ are the analytic functions and also are arising from the effect of compatibility equations
(Eq. (1-4)). The boundary conditions (Eqs. (68-75)) must be satisfied by the function of $\dot{u}_{1}\left(z-z_{1} \bar{z}\right)$ and $\dot{u}_{2}(z)$.

\section{Results and discussion}

3.1. Analyzing the behavior of $\dot{\boldsymbol{u}}_{\mathbf{1}}$. In the present work, displacement rates were determined by combination and solution of the compatibility, equilibrium, incompressibility, constitutive equations, strain and stress relations by complex variable method.

The behavior of the creeping fiber displacement rates $\dot{u}_{1}$ and $\dot{u}_{2}$ is graphically shown in Figs. 3a-d and $4 \mathrm{a}-\mathrm{d}$. The behavior of $\dot{u}_{1}$ in $3 \mathrm{D}$ coordinate with respect to $\bar{z}$ and $z$ for the low stresses and temperatures (constant $€$ ) is shown in Figs. 3a-d.

For example, linear behavior of $\dot{u}_{1}$ in 3D coordinate with respect to $\bar{z}$ and $z$ is shown in Fig. 3a. Moreover, Figs. 3a-d are presented for four different analytic functions $\rho(z)$.

According to Figs. $3 \mathrm{a}-\mathrm{d}$, it is concluded that the creep displacement rate behaviors based on the analytic functions of Figs. 3b, d are approximately correct and exact.

That is, selecting the even polynomial functions as the analytic functions yields the correct behavior in comparison with the odd functions of Figs. 3a, c. Also, Figs. 3b, d demonstrate a uniform behavior for $\dot{u}_{1}$, and also symmetric behavior is seen in these figures. In which, these symmetric behaviors in the diagrams prove the correct behavior of the creep (identical tensile and compressive creep deformations of unit cell due to the symmetric unit cell). Tensile deformations on the top and down of the fiber and matrix along with the compressive deformations on the left and right sides of the fiber and matrix are correct behaviors of the creep (see Figs. 3b, d).

Prediction of the creep behavior shown in Figs. 3b, $d$ is more accurate than the prediction presented in Figs. 3a, c. That is, more accurate results for predicting the creep behaviors are because of the choosing the even analytic polynomial functions for $\rho(z)$.

$\mathrm{T} \mid$ herefore, choosing odd analytic functions does not result in more accurate results comparing the even analytic polynomial functions for predicting the displacement rate $\dot{u}_{1}$.

3.2. Analyzing the behavior of $\dot{\boldsymbol{u}}_{\mathbf{2}}$. Here, the behaviors of $\dot{u}_{2}$ in $3 \mathrm{D}$ coordinate with respect to $\bar{z}$ and $z$ for the low stresses and temperatures (constant $€$ ) are presented in Figs. $4 \mathrm{a}-\mathrm{d}$.

In addition, the linear behavior of $\dot{u}_{2}$ in $3 \mathrm{D}$ coordinate with respect to $\bar{z}$ and $z$ is shown in Fig. $4 d$. Moreover, Figs. $4 \mathrm{a}-\mathrm{d}$ are presented for 4 analytic functions of $\rho(z)$.

In accordance with Figs. $4 \mathrm{a}-\mathrm{d}$, it is found that the creep displacement rate behaviors based on the analytic polynomial functions of Figs. b, c are approximately accurate and correct. That is, choosing the even polynomial functions as the analytic functions shows the correct behavior in comparison with the odd functions of Figs. a, d. In addition, Figs. 3b, c show a uniform behavior for $\dot{u}_{1}$, and also symmetric behavior is seen in these figures. These symmetric behaviors in the diagrams show the correct behavior of the creep. Tensile deformations on the top and down of the fiber and matrix along with the compres- 
a)

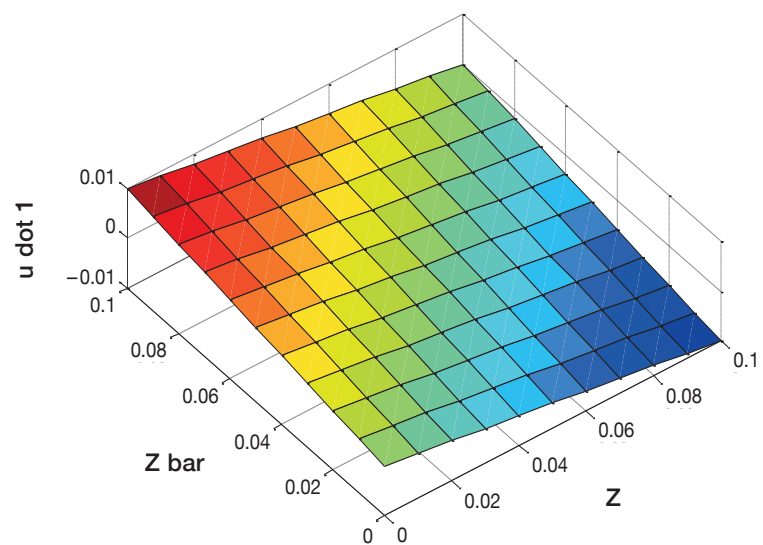

b)

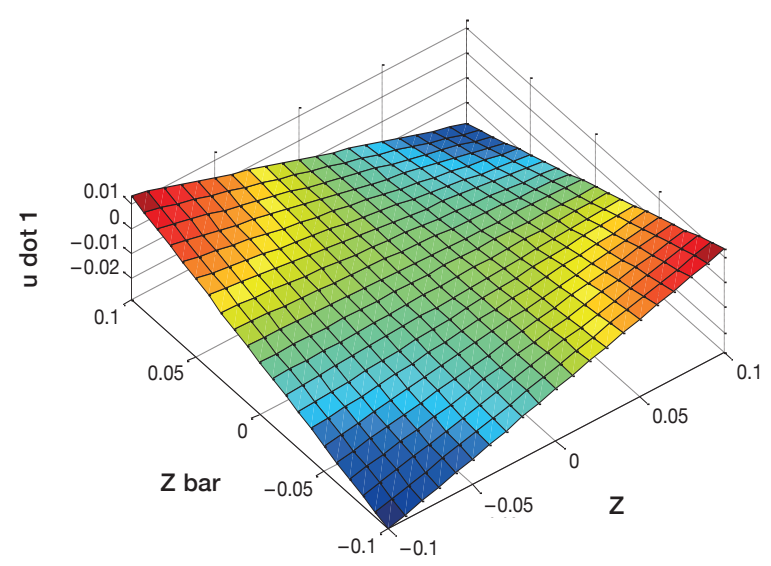

c)

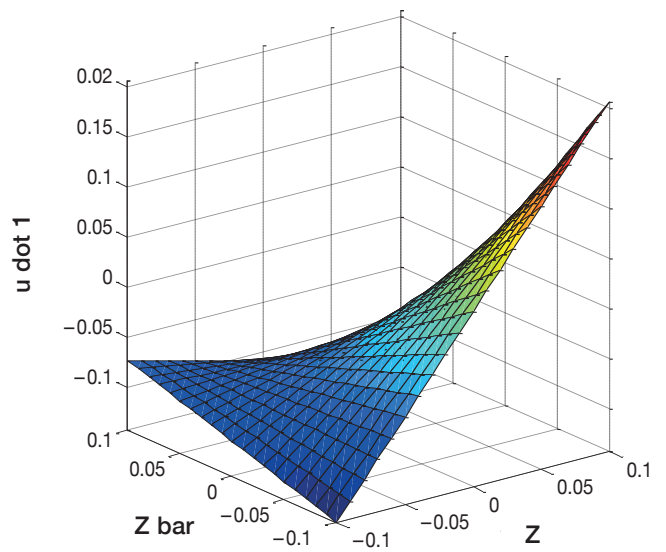

d)

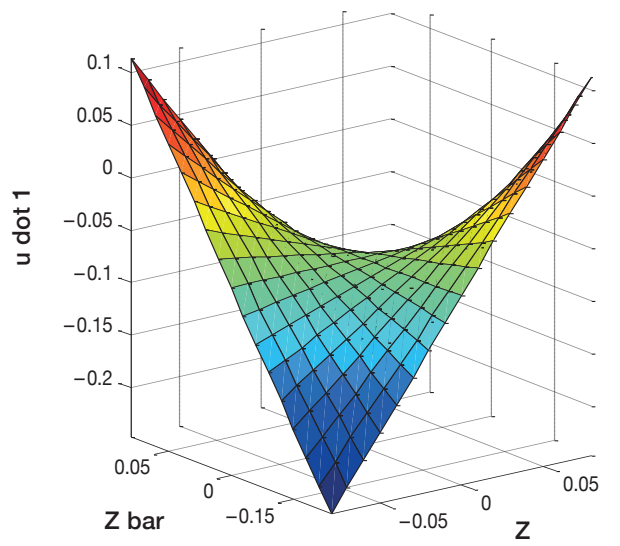

Fig. 3. a - Linear behavior of $\dot{u}_{1}$ with respect to $\bar{z}, z$ for $\rho(z)=z \cos (z)$, $\mathrm{b}$ - Nonlinear behavior of $\dot{u}_{1}$ with respect to $\bar{z}, z$ for $\rho(z)=z^{2}, \mathrm{c}-$ Nonlinear changes of $\dot{u}_{1}$ with respect to $\bar{z}, z$ for $\rho(z)=\operatorname{Sin}(z), \mathrm{d}$ - Linear behavior of $\dot{u}_{1}$ with respect to $\bar{z}, z$ for $\rho(z)=z^{4}$ a)

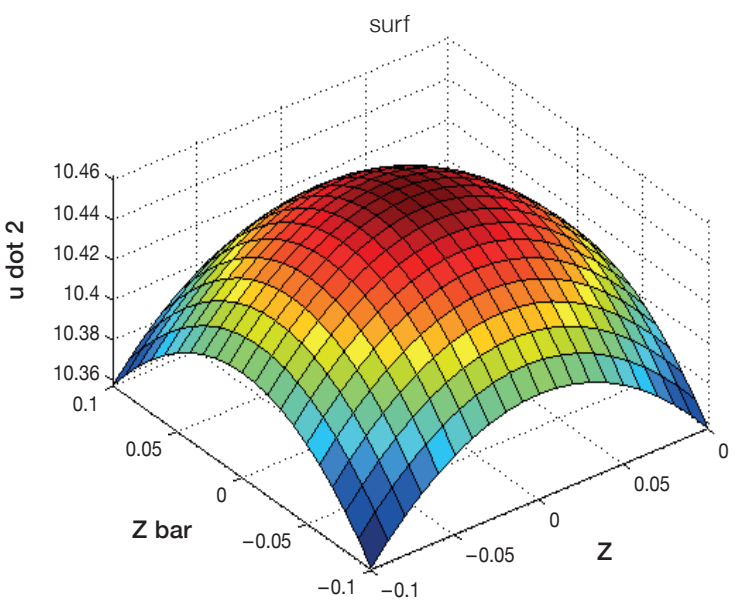

b)

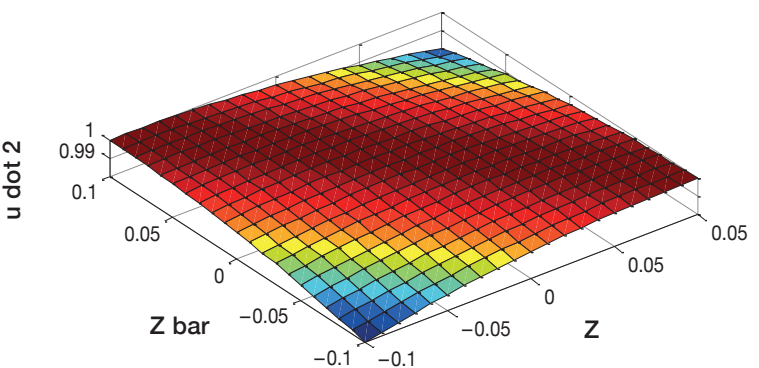

c)

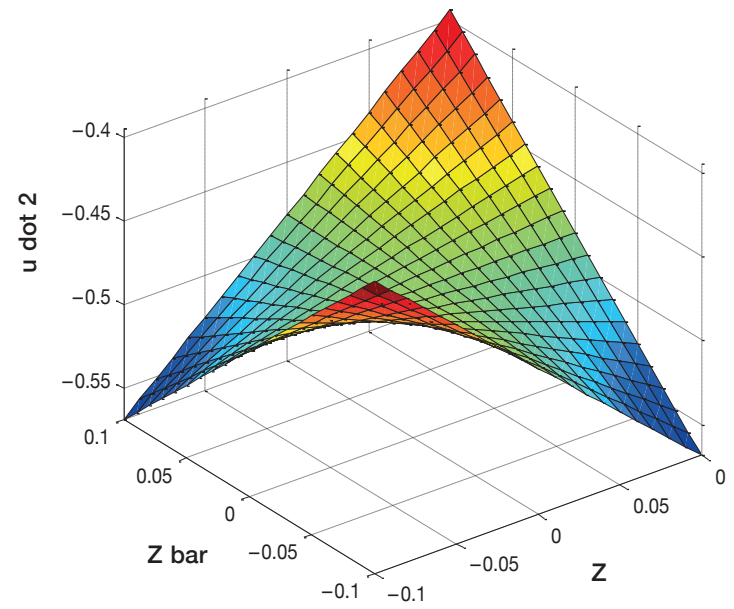

d)

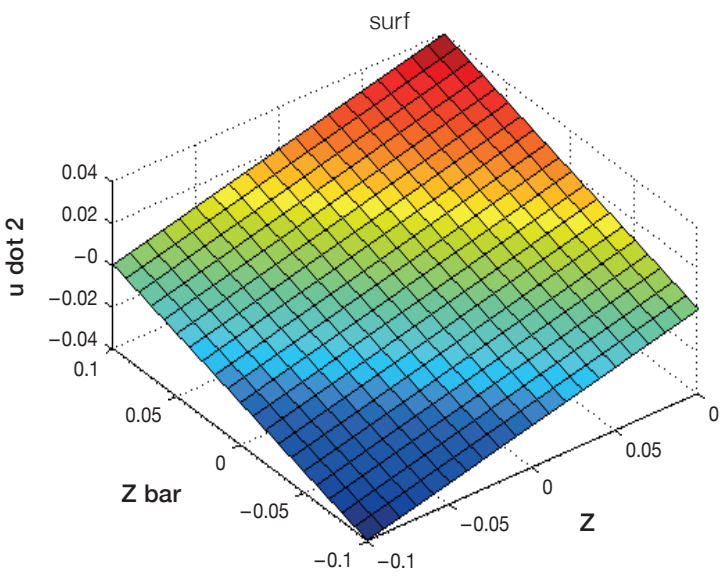

Fig. 4. a - Nonlinear changes of $\dot{u}_{2}$ with respect to $\bar{z}, z$ for $\rho(z)=z \cos (z)$, b - Nonlinear behavior of $\dot{u}_{2}$ with respect to $\bar{z}, z$ for $\rho(z)=z^{2}, \mathrm{c}$ - Nonlinear changes of $\dot{u}_{2}$ with respect to $\bar{z}, z$ for $\rho(z)=z^{4}, \mathrm{~d}-$ Nonlinear changes of $\dot{u}_{2}$ with respect to $\bar{z}, z$ for $\rho(z)=\operatorname{Sin}(z)$ 
sive deformations on the left and right sides of the fiber and matrix are acceptable behaviors of the creep (see Figs. 3b, c).

Prediction of the creep behavior shown in Figs. 3b, $\mathrm{c}$ is more accurate than the prediction presented in Figs. 3a, d. That is, more accurate results for predicting the creep behaviors are brought by choosing the even analytic polynomial functions for $\rho(z)$.

Thus, selecting the odd analytic functions did not yield more accurate results in comparison with the even analytic polynomial functions for predicting the displacement rate $\dot{u}_{2}$.

\section{Summary and conclusion}

In present work, a new complex variable method was presented for analyzing the steady state creep behavior in the short fiber composites, considering two cases for $€$ (constant and variable). On the other hand, displacement rate behaviors are predicted by means of compatibility, equilibrium, constitutive, governing, and stress-strain relations by complex variable method in the plane stress state. Partial differential equations were solved in the second stage creep in the short fiber composites by complex variable method and assumption of the plane stress state. Therefore, the creep problems may be solved and analyzed by change of space from Cartesian or polar to the complex plane simply. It is concluded that selecting the even polynomial functions for analytic function of $\rho(z)$ are more suitable than the odd functions for predicting the accurate displacement rate behavior of the fiber. Finally, some of the complicated problems can be solved by using complex variable method and dividing them into real and imaginary parts in the mechanical engineering and physics.

\section{REFERENCES}

[1] N.I. Muskhelishvili, Some Basic Problems of the Mathematical Theory of Elasticity, 4th ed., Moscow: Noordhoff, 1954.

[2] L.M. Milne-Thomson, Plane Elastic Systems, 1st ed., Berlin: Springer Verlag, 1960.

[3] A.E. Green and W. Zerna, Theoretical Elasticity, 2nd ed., London: Oxford University Press, 1968

[4] A.H. England, Complex Variable Methods in Elasticity, 1st ed., New York: John Wiley, 1971.

[5] X.L. Gao, "Alternative derivation of Marguerre's displacement solution in plane isotropic elasticity", ASME J. Appl. Mech. 67, 419-421 (2000).

[6] P. Barai and G.J. Weng, "A micro-continuum model for the creep behavior of complex nanocrystalline materials", Int. J. Eng. Sci. 49 (1), 155-174 (2011)

[7] T. Tang, M.F. Horstemeyer, and P. Wang, "Micromechanical analysis of thermoelastoplastic behavior of metal matrix composites", Int. J. Eng. Sci. 51, 161-167 (2012).
[8] Y. Li and Z. Li, "Transverse creep and stress relaxation induced by interface diffusion in unidirectional metal matrix composites", Compos. Sci. Technol. 72, 1608-1612 (2012).

[9] A. Loghman and V. Atabakhshian, "Semi-analytical solution for time-dependent creep analysis of rotating cylinders made of anisotropic exponentially graded material (EGM)", J. Solid. Mech. 4, 313-326 (2012).

[10] E. Hamed and Z.T. Chang, "Effect of creep on the edge debonding failure of FRP strengthened RC beams - A theoretical and experimental study", Compos. Sci.Technol. 74, 186-193 (2013).

[11] V. Monfared, S. Daneshmand and J.N. Reddy, "Rate dependent plastic deformation analysis of short fiber composites employing virtual fiber method", J. Comput. Sci. 10, 26-35 (2015).

[12] V. Monfared, S. Daneshmand, and A.H. Monfared, "Effects of atomic number and atomic weight on inelastic time dependent deformations", Kovov. Mater. 53 (2), 85-89 (2015).

[13] Martin H. Sadd, Elasticity: Theory, Applications, and Numerics, 3rd Edition, Academic Press, 2014.

[14] P.K. Duttaa and D. Hui, "Creep rupture of a GFRP comoposite at elevated temperatures", Comput Struct, 2000, 76 153-161 (2000).

[15] M. Hongyin and M. Sankaran, "Probabilistic analysis of creep of metal-matrix composites", J. Reinf. Plast. Compos. 21(7), 587-602 (2002).

[16] M. Sankaran and M. Hongyin, "Probabilistic fatigue-creep life prediction of composites", J. Reinf. Plas. Compos. 23(4), 361-371 (2004).

[17] J.R. Pachalis and T.W. Chou, "Modeling of creep of misaligned short-fiber reinforced ceramic composites", J. Appl. Mech, 59(1), 27-32 (1992).

[18] G.V. Kolosov, "On the application of complex function theory to a plane problem of the mathematical theory of elasticity", 1st ed. Moscow: Yuriev University Press, 1909.

[19] A. Zbiciak, "Mathematical description of rheological properties of asphalt-aggregate mixes", Bull. Pol. Ac.: Tech, 61 (1), 65-72 (2013).

[20] G. Leonardi, "Finite element analysis for airfield asphalt pavements rutting prediction", Bull. Pol. Ac.: Tech, 63 (2), 397-403 (2015).

[21] V. Monfared, "A micromechanical creep model for stress analysis of non-reinforced regions of short fiber composites using imaginary fiber technique", Mech. Mater. 86, 44-54 (2015).

[22] K.H. Grote and E.K. Antonsson, Handbook of Mechanical Engineering, Springer, 108-115, 2009.

[23] J.T. Boyle and J. Spence, Stress Analysis for Creep, 1st ed. Butterworth-Heinemann, Southampton: Butterworth, UK, 1983.

[24] Z. Chen, C. Majidi, D.J. Srolovitz, and M. Haataja, "Continuum elasticity theory approach for spontaneous bending and twisting of ribbons induced by mechanical anisotropy", arXiv preprint arXiv:1209.3321 (2012).

[25] A.K. Gaharwar, A. Patel, A. Dolatshahi-Pirouz, H. Zhang, K. Rangarajan, G. Iviglia, S.-R. Shin, M.A. Hussain, and A. Khademhosseini, "Elastomeric nanocomposite scaffolds made from poly (glycerol sebacate) chemically crosslinked with carbon nanotubes", Biomater. Sci., 3, 46-58. doi 10.1039/C4BM00222A (2015) 